\title{
Universiteit
}

Leiden

The Netherlands

\section{Improving Quality of Life in Depressed COPD Patients: Effectiveness of a Minimal Psychological Intervention}

Lamers, F.; Jonkers, C.C.M.; Bosma, H.; Chavannes, N.H.; Knottnerus, J.A.; Eijk, J.T. van

\section{Citation}

Lamers, F., Jonkers, C. C. M., Bosma, H., Chavannes, N. H., Knottnerus, J. A., \& Eijk, J. T. van. (2010). Improving Quality of Life in Depressed COPD Patients: Effectiveness of a Minimal Psychological Intervention. Copd: Journal Of Chronic Obstructive Pulmonary Disease, 7(5), 315-322. doi:10.3109/15412555.2010.510156

Version: $\quad$ Not Applicable (or Unknown)

License: $\quad$ Leiden University Non-exclusive license

Downloaded from: https://hdl.handle.net/1887/117621

Note: To cite this publication please use the final published version (if applicable). 


\title{
Improving Quality of Life in Depressed COPD Patients: Effectiveness of a Minimal Psychological Intervention
}

\author{
Femke Lamers ${ }^{1}$ (f.lamers@ vumc.nl), Catharina C.M. Jonkers ${ }^{1}$ (jonkers@ zonmw.nl), Hans Bosma ${ }^{1}$ \\ (hans.bosma@ socmed.unimaas.nl), Niels H. Chavannes ${ }^{2}$ (n.h.chavannes@lumc.nl), J. André Knottnerus ${ }^{3}$ \\ (andre.knottnerus@ hag.unimaas.nl), and Jacques ThM. van Eijk $\mathbf{1}^{\mathbf{1}}$ (j.vaneijk@ socmed.unimaas.nl) \\ ${ }^{1}$ School for Public Health and Primary Care (CAPHRI), Department of Social Medicine, Maastricht University, P.O. Box 616, 6200 MD, \\ Maastricht, the Netherlands \\ ${ }^{2}$ Department of Public Health and Primary Care, Leiden University Medical Center, P.O. box 9600, 2300 RC, Leiden, the Netherlands \\ ${ }^{3}$ School for Public Health and Primary Care (CAPHRI), Department of General Practice, Maastricht University, P.O. Box 616, 6200 MD, \\ Maastricht, the Netherlands
}

\begin{abstract}
Depression and anxiety are highly prevalent in elderly COPD patients. Since symptoms of depression and anxiety reduce quality of life in these patients, treatments aimed at improving mental health may improve their quality of life. This study evaluated the effectiveness of a nurse-led Minimal Psychological Intervention (MPI) in reducing depression and anxiety, and improving disease-specific quality of life in elderly COPD patients. In a randomized controlled trial an MPI was compared with usual care in COPD patients. COPD patients aged 60 years or over, and with minor or mild to moderate major depression were recruited in primary care (n $=187$ ). The intervention was based on principles of cognitive behavioural therapy (CBT) and self-management. Outcomes were symptoms of depression, symptoms of anxiety, and diseasespecific quality of life, assessed at baseline and at one week and three and nine months after the intervention. Results showed that patients receiving the MPI had significantly fewer depressive symptoms (mean BDI difference $2.92, p=0.04$ ) and fewer symptoms of anxiety (mean SCL difference $3.69, p=0.003$ ) at nine months than patients receiving usual care. Further, mean SGRQ scores were significantly more favourable in the intervention group than in the control group after nine months (mean SGRQ difference 7.94, $p=0.004$ ). To conclude, our nurse-led MPI reduced symptoms of depression and anxiety and improved disease-specific quality of life in elderly COPD patients. The MPI appears to be a valuable addition to existing diseasemanagement programmes for COPD patients.
\end{abstract}

Keywords: COPD, depression, aged, $C B T$, self management, Quality of life

Funding: This study was funded by the Netherlands Organisation for Health Research and Development (ZonMw), programme on Health Care Efficiency Research, grant number 945-03-047. Correspondence to:

F. Lamers

VU Medical Center

Department of Psychiatry

AJ Ernststraat 887

1081 HL Amsterdam

phone: +31207884583

fax: +31207885664

email: f.lamers@vumc.nl

\section{INTRODUCTION}

Chronic obstructive pulmonary disease (COPD) is a prevalent disease in elderly persons (1). Since COPD is irreversible, disabling and progressive, treatment aims to prevent deterioration of the disease and maintain a high quality of life.

Depression and anxiety are highly prevalent in elderly COPD patients $(2,3)$, and both are known to reduce quality of life (4-6). Recent research has shown that depressive symptoms are strongly associated with worse quality of life, even after severity of COPD was taken into account (7). The exact causal mechanisms for depression in COPD are not well known, making it hard to select therapeutic targets. However, since COPD is characterized by systemic inflammation (8), and depression is known to have an adverse influence on immunologic processes 
$(9,10)$, co-occurrence of COPD and depression may lead to further deterioration of patients' health status due to interaction. Breaking through this downward spiral may thus be an important step towards improving quality of life. In view of the relationship between quality of life and depression and anxiety, treatments to reduce depression and anxiety in COPD patients may also improve their quality of life $(3,11)$.

Depression and anxiety can be treated with antidepressants $(3,12,13)$, although some claim that the evidence for their effectiveness in older COPD patients is sparse and inconclusive $(12,14)$. Furthermore, treatment with antidepressants may be complicated by problems like medication side effects or refusal of treatment by COPD patients $(12,14)$. Psychological and selfmanagement interventions may be better alternatives, especially as they teach patients valuable and enduring skills to cope with their disease, thus potentially ensuring a more lasting effect, extending beyond the end of treatment. Furthermore, these interventions can be administered by trained paramedical professionals, like nurses, complementing the care of physicians (15, 16). Few trials have assessed the effectiveness of psychological and self-management interventions in elderly COPD patients in terms of reducing symptoms of depression and anxiety. A recent systematic review based on four, relatively small, studies, found only limited evidence for the effectiveness of cognitive behavioural therapy (CBT) in reducing anxiety and depression in COPD patients, although the direction of treatment effects on both depression and anxiety scales mainly favoured CBT. (17) The largest trial to date on CBT on COPD is a study by Kunik and colleagues (18). This study showed that CBT as well as COPD education improved quality of life, depression and anxiety symptoms, among persons with high scores on inventories for anxiety and depression symptomatology. A recent review of self-management education for COPD patients produced inconclusive results for the effect on the generic quality of life outcome, but found significant effects on a disease-specific quality of life measure (19). Both reviews recommend further research into CBT and self-management for COPD patients.

We have developed a nurse-administered minimal psychological intervention (MPI) based on the principles of CBT and self-management. The intervention has previously been evaluated in the Depression in Elderly with long-Term Afflictions (DELTA) study in a population of elderly patients with diabetes or COPD (20). The current contribution reports on the effect of the MPI on disease-specific quality of life and symptoms of depression and anxiety in the subgroup of elderly COPD patients with co-morbid depression. We hypothesized that our MPI would reduce symptoms of depression and anxiety and would also improve disease-specific quality of life.

\section{MATERIALS \& METHODS}

\section{Study design}

Detailed information on the study protocol has been published elsewhere $(20,21)$. Briefly, a randomized controlled trial was conducted, with patients allocated to either the MPI or care as usual after signing informed consent. Self-administered questionnaires were used to collect data at baseline and at one week and three and nine months after the treatment period. Data entry was performed by researchers blinded for the allocation. Approval for this study was obtained from the Medical Ethics Committee of Maastricht University / University Hospital Maastricht.

\section{Participants and procedures}

Between October 2003 and May 2005, patients were recruited in 89 general practices in the south of the Dutch province of Limburg. We first selected all patients aged 60 years and older with an International Classification of Primary Care (ICPC) code for emphysema / COPD or chronic bronchitis. If ICPC codes were not available, lists of patients with repeat prescriptions for bronchodilatory drugs were obtained. The patients' general practitioners (GPs) then excluded from this initial broad selection all patients who did not have COPD, were bedridden, were on a waiting list for a nursing home, had major depression, used antidepressants, had major psychiatric conditions or were currently receiving psychosocial/psychiatric treatment, had serious cognitive problems, had recently lost their spouse or were not fluent in Dutch. All remaining patients aged 60 years and older who did have COPD according to their GP were sent a depression screening questionnaire (Patient Health Questionnaire-9 PHQ-9) (22). After the PHQ-9 screening, all patients who reported having at least two symptoms of depression, one being loss of interest or depressed mood, were invited to a diagnostic interview, consisting of the Mini International Neuropsychiatric Interview (MINI) (23) and the Hamilton Depression Rating Scale (HDRS) (24). The interview was conducted at the patients' home by a trained nurse. Patients with minor depression, mild major depression, moderate major depression or dysthymia were included in the study. Patients with severe major depression according to the HDRS (HDRS > 18) and patients with suicidal risk were excluded and referred back to their GP. All COPD patients thus included $(n=187)$ signed an informed consent form and were randomized. The researchers entered patients into a computer connected to an external agency, which performed the randomization using a computerized random number generator. A block randomization scheme was used, stratified for general practice. Block size was set at 2, as we expected to include only a small number of patients per practice.

\section{Intervention and usual care}

Patients allocated to the intervention group received the MPI at home, supplementary to usual care according to the clinical Guidelines for the Treatment of COPD of the Dutch College of General Practitioners (25). The intervention is a nurseadministered, minimal psychological intervention, consisting of elements of CBT and self-management. Nurses were trained by a GP, a psychologist and a psychiatrist, and had regular booster sessions with the psychiatrist. The intervention was tailored to individual patients. Depending upon progress, patients received two to ten visits over a period of at most three months. 


\begin{tabular}{ll}
\hline Table 1. Phases of the Minimal Psychological Intervention (MPI) \\
\hline Phase & Description \\
1 & $\begin{array}{l}\text { The nurse explores the patient's feelings, cognitions and behaviours } \\
\text { The patient keeps a diary, where he or she records symptoms, complaints, thoughts, worries, related feelings and } \\
\text { behaviour } \\
\text { The patient is challenged to link his or her mood to the consequent behaviour, using information from the diary } \\
\text { The self-management approach is introduced. The patient explores possibilities to alter his or her behaviour and draws } \\
\text { up an action plan }\end{array}$ \\
5 & Evaluation of progress in achieving the goals of the action plan \\
\hline
\end{tabular}

Patients had on average four intervention contacts, each lasting approximately one hour. Table 1 lists the five phases of the intervention; a more detailed description of the intervention has been published elsewhere $(21,26)$. To ensure that the nurses adhered to the protocol during the study, they were asked to keep checklists, covering all essential intervention steps, for each patient. Examination of these lists showed that nurses had adhered closely to the guidelines in the protocol (27). Patients allocated to the control group received care as usual according to the above-mentioned clinical guidelines.

\section{Outcome measures}

Data were collected by means of self-administered questionnaires. Depressive symptoms were assessed using the Beck Depression Inventory (BDI) (28) and anxiety was assessed using the anxiety subscale of the Symptom Checklist-90 (SCL) (29). Demographic information was collected at the time of the PHQ9 screening. Disease-specific quality of life was assessed using the Saint George's Respiratory Questionnaire (SGRQ) (30). The SGRQ consists of a total score that summarizes the overall impact of COPD on health status, and three subscales: the activity scale (activities that cause or are limited by breathlessness), the impact scale (social functioning and psychological disturbances) and the symptom scale (effects, frequency and severity of respiratory symptoms). Missing item scores on the SGRQ and SCL were imputed according to the recommendations in their respective manuals; missing items on the BDI were imputed with the patient's mean item score as long as at least $50 \%$ of the items had been completed.

\section{Statistical analysis}

The power calculation for the DELTA study was based on the BDI in the total group of diabetes and COPD patients (20). According to this calculation, assuming an $\alpha$ of 0.05 and a $1-\beta$ (power) of 0.90 , we needed to include $2 \times 96=192$ patients (48 COPD and $48 \mathrm{DM}$ patients in the intervention group and $48 \mathrm{COPD}$ and $48 \mathrm{DM}$ patients in the control group) to allow us to detect a minimum clinically relevant difference of $18 \%$ in improvement $(\geq 50 \%$ reduction of BDI score relative to baseline). (31) Anticipating an attrition rate of approximately $30 \%$ and the potential need for subgroup analyses, we decided to recruit a gross number of 360 patients. In the group of COPD patients included in the current study $(\mathrm{n}=187)$, a difference of 7.83 on the SGRQ total score would be significant at the $5 \%$ level. A difference of four points on the SGRQ is considered clinically relevant.

Analyses were based on the intention-to-treat principle. The comparability of groups was checked by means of t-tests and Chi-square tests for demographic and outcome variables. Mixed model, repeated-measure ANCOVA analysis was used to test the differences between groups at follow-up measurements. Advantages of mixed model analysis include that it can handle missing observations, and error terms are estimated more precisely, thereby further increasing power. Fixed effects included in the model were: age, gender, educational level, treatment group, baseline value of outcome, time and the product term of time and group. Several random effects and covariance matrices were tested, using -2 log likelihood tests to decide which model had the best fit. Maximum Likelihood models were then run, whose results are presented here.

In addition, effect sizes (d) were calculated by dividing the difference in mean group scores by the pooled standard deviation (32). The percentage of patients showing an improvement of 4 points on the SGRQ total score was also computed and differences between groups were tested with Chi-square tests.

Additional per-protocol analyses were done, including patients who had received a complete intervention (all core steps of the intervention delivered, based on nurses' checklists, $\mathrm{n}=$ 60 (27)) and excluding 4 control patients who had received information on the content of the intervention and had benefited from this (based on self-report). We also performed analyses in which missing observations were imputed (last observation carried forward - LOCF).

\section{RESULTS}

Figure 1 shows the flow of COPD patients in the trial. Of the 506 patients invited for a MINI, 164 did not meet the inclusion criteria. The 41 patients who were eligible but refused to participate were significantly older than patients who did enter the study. Of the 187 patients who were included, 96 were offered the MPI, and 91 received usual care. The dropoutrate was $36 \%$, which was slightly higher than the anticipated $30 \%$. Although the difference was not statistically significant, the dropout-rate was somewhat higher in the intervention group than in the control group ( $40 \%$ vs. $33 \%, \mathrm{p}=0.35$ ). Furthermore, dropouts overall had a higher age and higher baseline BDI and SGRQ scores (activity and impact subscales and total score), 
Excluded $(n=319)$

Not participating in MINI:

Refused to participate in MINI or could not be reached $(n=114)$

Participating in MINI:

Not meeting inclusion criteria $(n=164)$

Refused to participate in trial $(n=41)$

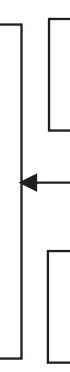

Invited for MINI ( $\mathrm{n}=506)$

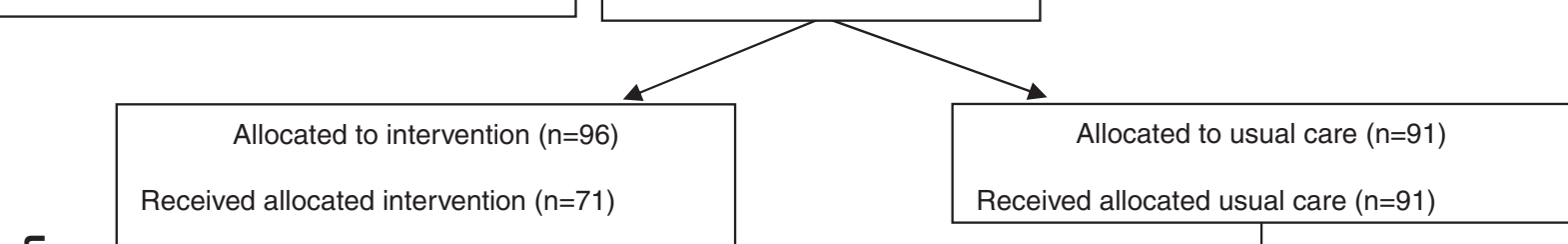

흘

Discontinued intervention $(n=16)$

Lack of perceived usefulness $(n=5)$

Perceived burden $(n=3)$

Personal/family circumstances $(n=1)$

Physical decline $(n=3)$

Cognitive decline $(n=1)$

Deceased $(n=2)$ Other reasons $(n=1)$

Did not receive allocated intervention $(n=9)$

Lack of perceived useful $(n=6)$

Physical decline $(n=2)$

Other reasons $(n=1)$

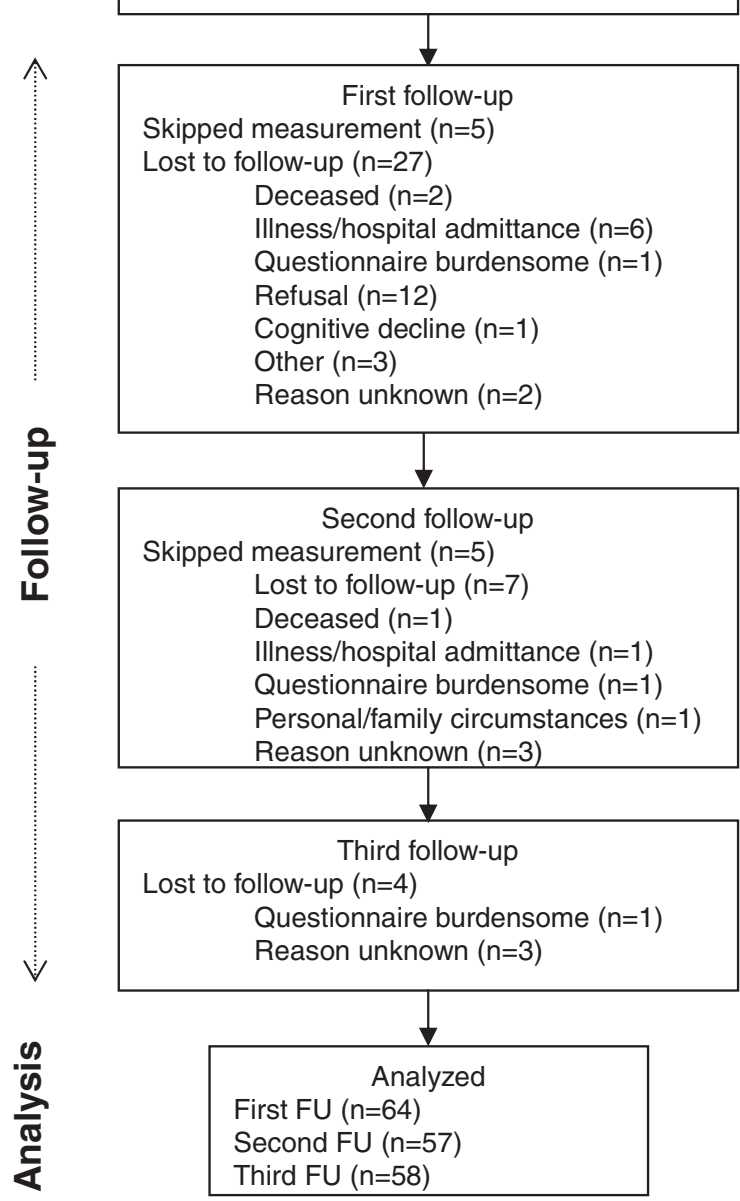

First follow-up

Skipped measurement $(n=6)$

Lost to follow-up $(n=17)$

Illness/hospital admittance $(n=6)$

Questionnaire burdensome $(\mathrm{n}=2)$

Refusal $(n=4)$

Personal/family circumstances $(n=1)$

Other $(n=1)$

Reason unknown $(\mathrm{n}=3)$

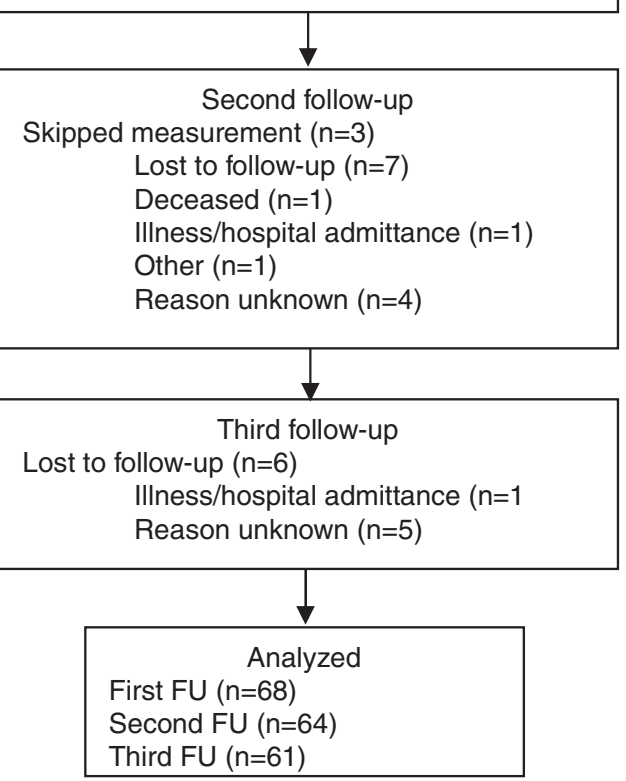

Figure 1. Patient flow and follow up in the study. 


\begin{tabular}{|c|c|c|c|}
\hline Variable & $\begin{array}{c}\text { Usual care } \\
n=91\end{array}$ & $\begin{array}{c}\text { MPI } \\
n=96\end{array}$ & P-value \\
\hline Age, yrs (SD) & $71.5(7.1)$ & $70.5(6.3)$ & 0.32 \\
\hline \multicolumn{4}{|l|}{ Sex, No. (\%) } \\
\hline Male & $53(58.2)$ & $59(61.5)$ & \\
\hline Female & $38(41.8)$ & $37(38.5)$ & 0.65 \\
\hline \multicolumn{4}{|l|}{ Education level ${ }^{\dagger}$, No. (\%) } \\
\hline Low & $31(34.1)$ & $36(37.5)$ & \\
\hline Medium & $24(26.4)$ & $31(32.3)$ & \\
\hline High & $36(39.6)$ & $29(30.2)$ & 0.39 \\
\hline $\mathrm{BDI}$, mean (SD) & $18.3(7.2)$ & $17.1(6.5)$ & 0.23 \\
\hline SCL, mean (SD) & $20.4(7.3)$ & $20.6(6.2)$ & 0.83 \\
\hline SGRQ total score, mean (SD) & $56.8(19.5)$ & $54.9(17.5)$ & 0.55 \\
\hline SGRQ activity, mean (SD) & $70.9(21.0)$ & $70.6(20.9)$ & 0.79 \\
\hline SGRQ impact, mean (SD) & $46.2(22.3)$ & $45.9(20.8)$ & 0.91 \\
\hline SGRQ symptoms, mean (SD) & $56.7(27.5)$ & $60.9(25.2)$ & 0.30 \\
\hline \multicolumn{4}{|c|}{$\begin{array}{l}\dagger \text { Low refers to primary school only, medium refers to lower vocational training or lower general education, high refers } t \\
\text { higher vocational training, secondary school, higher professional education and university training. } \\
\text { Range of the BDI is } 0-63 \text {, with } 0 \text { as the most favorable outcome; range of SGRQ total scale and subscales is } 0-100 \text {, } \\
\text { with } 0 \text { as the most favorable score; range of the SCL Anxiety scale is } 1-50 \text {, with } 1 \text { as the most favorable outcome. }\end{array}$} \\
\hline
\end{tabular}

indicating worse depression states and lower quality of life (data not shown).

Table 2 shows the comparability of groups at baseline. None of the differences between the intervention and control groups were statistically significant, although the control group was slightly older and had a somewhat higher educational level than the intervention group. In addition, the control group had somewhat higher mean BDI and SGRQ total scores, indicating a higher level of depressive symptoms and lower quality of life than the intervention group.

Results of the mixed model analyses are presented in Table 3. On average, we found significantly lower scores for both the depression and anxiety outcomes at nine months in the intervention group compared with the control group, indicating fewer symptoms (BDI $\mathrm{p}=0.04$; SCL $\mathrm{p}=0.003$ ). As for diseasespecific quality of life, we found that the intervention group had significantly better scores on the SGRQ activity subscale than controls at one week $(\mathrm{p}=0.004)$ and three months $(\mathrm{p}=$ 0.02) after the intervention, but the mean difference between groups was no longer significant at nine months after the intervention ( $\mathrm{p}=0.09$ ). Results for the SGRQ impact subscale showed the opposite pattern: there were no significant differences immediately after the intervention, but the intervention group had significantly better scores than the control group at three months $(p=0.02)$ and nine months $(p=0.003)$ after the intervention. No effects were found on the SGRQ symptom subscale, but the SGRQ total scores showed that, on average, intervention patients had a significantly better quality of life than controls at all follow-up measurements $(1 \mathrm{wk} \mathrm{p}=0.03 ; 3$ months $\mathrm{p}=0.005 ; 9$ months $\mathrm{p}=0.004)$. In terms of clinical relevance, a four-point change relative to baseline on the SGRQ total score was more often seen in intervention patients than in control patients at all follow-up measurements, with a signif- icant difference at nine months (52\% vs. $29 \%, \mathrm{p}=0.03$, not tabulated). Effect sizes corresponding to significant results can all be classified as medium. (32) Additional per-protocol and LOCF analyses produced similar results (data not shown).

\section{DISCUSSION}

This study showed that a nurse-led minimal psychological intervention significantly reduced depressive symptoms and prevented aggravation of anxiety in elderly COPD patients with minor to moderate depression. The intervention also improved disease-specific quality of life. Corresponding effect sizes were all medium (32).

Our tailor-made intervention was intended to educate patients to take responsibility for the day-to-day management of their own illness and its consequences. This was achieved by improving patients' understanding of the relation between their behaviour and consequent mood, by challenging them to draw up action plans to change their behaviour, and by teaching them self-management skills that they could apply in daily life. The study results indicate that the aim of the intervention was indeed achieved, as differences between groups became larger over time for all outcomes, except for the Activity subscale. This may very well mean that patients learned skills and continued to apply them in daily life during the follow-up period, even becoming more successful over time. Winning back control over daily symptoms by learning coping skills seems very rewarding especially for COPD patients, as many of them have developed a tendency towards 'learned helplessness' during the course of their disease. This explanation is further supported by the results of analyses showing positive effects on self-efficacy, which is a prerequisites for improvement of self-care. Also social participation appeared to be influenced positively (33). Also, improved 
regarded as a conservative imputation method (36), did not alter our conclusions. Second, disease severity can be an interfering factor if severity is unequally divided between groups and also effectivity of the intervention may differ between moderate and severe cases of COPD. We did not have objective data on the severity of COPD among participants at baseline, such as FEV1 data, since patient inclusion was done by GPs on the basis of existing practice records. However, disease-specific quality of life, which has been shown to be related to COPD severity but may reflect disease impact better (37), did not differ between groups at baseline. Also, recent research has shown that a postbronchodilator FEV1/FVC ratio of 0.7 was supported by SGRQ measurements (38), which may indicate that SGRQ scores may be used as surrogate markers of lung function. Third, we included persons who according to their GP had COPD, but nevertheless some persons may not have had a COPD diagnosis confirmed by spirometry. Fourth, blinding of patients was obviously not possible, as we compared the intervention with usual care, as is often done in pragmatic trials.

Our study had several strengths. Suspected depression, as picked up by the screening questionnaire, was confirmed by a DSM-IV based diagnostic interview. Additionally, we included patients with a wide range of depression severity, so that our sample reflects the natural heterogeneity of patients with depressive symptoms in primary care. A further strength of our approach is that nurses were used to administer the intervention. Since nurses are already involved in disease-management programmes and as such see patients on a regular basis, they seem obvious candidates for the task of administering psychological interventions. We have shown that nurses without any specific psychiatric background can be trained to administer an MPI that successfully reduces depression and anxiety and improves quality of life. An extensive process evaluation showed that nurses had adhered closely to the protocol and that they were highly enthusiastic about the intervention. As for patients, the evaluation revealed that they were also highly satisfied with the intervention and would recommend it to other persons with a chronic illness (27). Chronic disease management strategies are creating a growing burden on patients, which may lead to poor adherence, wasted resources and poorer outcomes (39). The outcomes of the process evaluation however, indicate that the MPI is very much appreciated by patients and was even recommended to be offered to all patients with chronic diseases. MPI therefore seems to be very suitable for incorporation within disease management programmes, which appears a logical next step. By taking on the task of depression management within these programmes, nurses may thus complement the GP's regular work. An economic evaluation of the intervention in the original study population (diabetes and COPD) showed a 63\% probability that the MPI is less costly and more effective than usual care, and it appeared that the intervention is no more costly than care as usual (40).

In a time when aging of the population is likely to increase the prevalence and burden of COPD, maintaining the highest possible quality of life and slowing down further deterioration of the COPD patient's health status will remain the key goals of
COPD treatment. Our nurse-administered minimal psychological intervention was effective in reducing anxiety and depressive symptoms and improving disease-specific quality of life. We therefore feel that adding MPI to existing disease-management programmes is likely to improve the care for elderly COPD patients.

\section{ACKNOWLEDGMENT}

We want to thank our nurses, Kitty Daemen, Henny Geelen, Francine Hendriks and Mieke Witte for administering the intervention with great dedication and enthusiasm, as well as Wendy Engering for her contribution to patient recruitment and Dorien Mintjes for her contribution to data entry, and MEMIC (Centre for Data and Information Management) for providing us with a data management system.

\section{Declaration of interest}

The authors report no conflicts of interest. The authors alone are responsible for the content and writing of the paper.

\section{REFERENCES}

1. Halbert RJ, Natoli JL, Gano A, Badamgarav E, Buist AS, Mannino DM. Global burden of COPD: systematic review and metaanalysis. Eur Respir J 2006; 28(3):523-532.

2. Kunik ME, Roundy K, Veazey C, Souchek J, Richardson P, Wray NP, Stanley MA. Surprisingly high prevalence of anxiety and depression in chronic breathing disorders. Chest 2005; 127(4):1205-1211.

3. Mikkelsen RL, Middelboe T, Pisinger C, Stage KB. Anxiety and depression in patients with chronic obstructive pulmonary disease (COPD). A review. Nord J Psych 2004; 58(1):65-70.

4. Gudmundsson G, Gislason T, Janson C, Lindberg E, Suppli Ulrik C, Brondum E, Nieminen MM, Aine T, Hallin R, Bakke P. Depression, anxiety and health status after hospitalisation for COPD: a multicentre study in the Nordic countries. Respir Med 2006; 100(1):87-93.

5. Hynninen KM, Breitve MH, Wiborg AB, Pallesen S, Nordhus IH. Psychological characteristics of patients with chronic obstructive pulmonary disease: a review. J Psychosom Res 2005; 59(6):429-443.

6. Yohannes AM, Baldwin RC, Connolly MJ. Prevalence of subthreshold depression in elderly patients with chronic obstructive pulmonary disease. Int J Geriatr Psych 2003; 18(5):412-416.

7. Omachi TA, Katz PP, Yelin EH, Gregorich SE, Iribarren C, Blanc PD, Eisner MD. Depression and health-related quality of life in chronic obstructive pulmonary disease. Am J Med 2009; 122(8):778e9-15.

8. Gan WQ, Man SF, Senthilselvan A, Sin DD. Association between chronic obstructive pulmonary disease and systemic inflammation: a systematic review and a meta-analysis. Thorax 2004; 59(7):574-580.

9. Maes M. Evidence for an immune response in major depression: a review and hypothesis. Prog Neuropsychopharmacol Biol Psych 1995; 19(1):11-38.

10. Herbert TB, Cohen S. Depression and immunity: a meta-analytic review. Psychol Bull 1993; 113(3):472-486.

11. Cully JA, Graham DP, Stanley MA, Ferguson CJ, Sharafkhaneh A, Souchek J, Kunik ME. Quality of life in patients with chronic obstructive pulmonary disease and comorbid anxiety or depression. Psychosomatics 2006; 47(4):312-319. 
12. Norwood R, Balkissoon R. Current perspectives on management of co-morbid depression in COPD. COPD 2005; 2(1):185-193.

13. Brenes GA. Anxiety and chronic obstructive pulmonary disease: prevalence, impact, and treatment. Psychosom Med 2003 65(6):963-970.

14. Yohannes AM, Baldwin RC, Connolly MJ. Depression and anxiety in elderly patients with chronic obstructive pulmonary disease. Age Ageing 2006; 35(5):457-459.

15. Hunkeler EM, Meresman JF, Hargreaves WA, Fireman B, Berman WH, Kirsch AJ, Groebe J, Hurt SW, Braden P, Getzell M, Feigenbaum PA, Peng T, Salzer M. Efficacy of nurse telehealth care and peer support in augmenting treatment of depression in primary care. Arch Fam Med 2000; 9(8):700-708.

16. Mynors-Wallis LM, Gath DH, Day A, Baker F. Randomised controlled trial of problem solving treatment, antidepressant medication, and combined treatment for major depression in primary care. BMJ 2000; 320(7226):26-30.

17. Coventry PA, Gellatly JL. Improving outcomes for COPD patients with mild-to-moderate anxiety and depression: A systematic review of cognitive behavioural therapy. $\mathrm{Br} \mathrm{J}$ Health Psychol 2008; 13(Pt 3):381-400.

18. Kunik ME, Veazey C, Cully JA, Souchek J, Graham DP, Hopko D, Carter R, Sharafkhaneh A, Goepfert EJ, Wray N, Stanley MA. COPD education and cognitive behavioral therapy group treatment for clinically significant symptoms of depression and anxiety in COPD patients: a randomized controlled trial. Psychol Med 2008; 38(3):385-396.

19. Effing T, Monninkhof EM, Van Der Valk PD, Van Der Palen J, van Herwaarden CL, Partidge MR, Walters EH, Zielhuis GA. Self-management education for patients with chronic obstructive pulmonary disease. Cochrane Database Syst Rev, 2007(4):CD002990.

20. Lamers F, Jonkers CCM, Bosma H, Kempen GIJM, Meijer JAMJ, Penninx BWJH, Knottnerus JA, van Eijk JTM. A minimal psychological intervention in chronically ill elderly patients with depression: A randomised trial. Psychother Psychosom, 2010. 79(4):217-226.

21. Lamers F, Jonkers CC, Bosma H, Diederiks JP, van Eijk JT. Effectiveness and cost-effectiveness of a minimal psychologica intervention to reduce non-severe depression in chronically ill elderly patients: the design of a randomised controlled trial [ISRCTN92331982]. BMC Public Health 2006; 6(1):161.

22. Spitzer RL, Kroenke K, Williams JB. Validation and utility of a self-report version of PRIME-MD: the PHQ primary care study. Primary Care Evaluation of Mental Disorders. Patient Health Questionnaire. JAMA 1999; 282(18):1737-1744.

23. Sheehan DV, Lecrubier Y, Sheehan KH, Janavs J, Weiller E, Keskiner A, Schinka J, Knapp E, Sheehan MF, Dunbar GC. The validity of the Mini International Neuropsychiatric Interview (MINI) according to the SCID-P and its reliability. Eur Psych 1997; 12(5):232-241.

24. Hamilton M. A rating scale for depression. J Neurol Neurosurg Psych 1960; 23:56-62.

25. Geijer RMM, Thiadens HA, Smeele IJM, Sachs APE, Bottema BJAM, van Hensbergen W, van Schayk CP, van Weel C, Rosmalen CFH. NHG-Standaard COPD en Astma bij Volwassenen:
Diagnostiek [NHG Practice Guideline COPD: Diagnosis]. Huisarts Wet 2001; 44(3):107-117.

26. Van Eijk JT, Diederiks JP, Kempen GI, Honig A, Van Der Meer K, Brenninkmeijer WJ. Development and feasibility of a nurse administered strategy on depression in community-dwelling patients with a chronic physical disease. Patient Educ Couns, 2004. 54(1): 87-94.

27. Jonkers CCM, Lamers F, Bosma H, Metsemakers JF, Kempen GIJM, van Eijk JTM. Process evaluation of a minimal psychological intervention to reduce depression in chronically ill elderly persons. Patient Educ Couns 2007; 68(3):252-257.

28. Beck AT, Ward CH, Mendelson M, Mock J, Erbaugh J. An inventory for measuring depression. Arch Gen Psych 1961; 4:561-571.

29. Arrindel WA, Ettema JHM. SCL-90: Handleiding bij een multidimensionele psychopathalogie-indicator. 1986, Lisse: Swets en Zeitlinger B.V.

30. Jones PW, Quirk FH, Baveystock CM, Littlejohns P. A selfcomplete measure of health status for chronic airflow limitation. The St. George's Respiratory Questionnaire. Am Rev Respir Dis 1992; 145(6):1321-1327.

31. Pocock S. Clinical trials: A Practical Approach. Chichester: John Wiley and Sons; 1983,

32. Cohen J. Statistical power analysis for the behavioral sciences. 2nd ed., Hillsdale, NJ: Lawrence Erlbaum Associates; 1988.

33. Jonkers CCM, Lamers F, Bosma H, Metsemakers JFM, Van Eijk JTM. The impact of a minimal psychological intervention on daily functioning, self-efficacy, mastery, autonomy, and anxiety in chronically ill elderly patients: findings from a randomized trial (the DELTA study). (submitted).

34. Jones PW. St. George's Respiratory Questionnaire: MCID. COPD 2005; 2(1):75-79.

35. Puhan MA, Guyatt GH, Goldstein R, Mador J, McKim D, Stahl E, Griffith L, Schunemann HJ. Relative responsiveness of the Chronic Respiratory Questionnaire, St. Georges Respiratory Questionnaire and four other health-related quality of life instruments for patients with chronic lung disease. Respir Med 2007; 101(2):308-316.

36. Beunckens $\mathbf{C}$, Molenberghs $\mathbf{G}$, and Kenward MG, Direct likelihood analysis versus simple forms of imputation for missing data in randomized clinical trials. Clin Trials 2005; 2(5):379386.

37. Stahl E, Lindberg A, Jansson SA, Ronmark E, Svensson K, Andersson F, Lofdahl CG, Lundback B. Health-related quality of life is related to COPD disease severity. Health Qual Life Outcomes 2005; 3:56.

38. Weatherall M, Marsh $S$, Shirtcliffe $P$, Williams $M$, Travers J, Beasley R. Quality of life measured by the St George's Respiratory Questionnaire and spirometry. Eur Respir J 2009; 33(5):1025-1030.

39. May C, Montori VM, and Mair FS. We need minimally disruptive medicine. BMJ 2009; 339:b2803.

40. Jonkers CC, Lamers F, Evers SM, Bosma H, Metsemakers JF, Van Eijk JT. Economic evaluation of a minimal psychological intervention in chronically ill elderly patients with minor or mild to moderate depression: a randomized trial (the DELTA-study). Int $J$ Technol Assess Health Care 2009; 25(4):497-504. 